\title{
UNIQUE COMMON FIXED POINTS FOR GENERALIZED CONTRACTIVE TYPE MAPPINGS IN $D_{1}$-SPACE
}

\author{
Yamin Wang ${ }^{1}$, Linan Zhong ${ }^{2} \S$ \\ ${ }^{1,2}$ Department of Mathematics \\ Yanbian University \\ P.R. Yanji, 133000, P.R. CHINA
}

$\mathrm{g}$

\begin{abstract}
In this paper we introduce a new notion, named as $D_{1}$-space, which is weak than $D$-metric space and $G$-metric space. Then several families of generalized contractive type mappings are defined on the $D_{1}$-space. We then obtain some theorems on the existence of unique common fixed points for these mappings which additionally satisfy the converse commuting condition.
\end{abstract}

AMS Subject Classification: 54H25, 47H10

Key Words: $D_{1}$-space, fixed point, converse commuting self-maps

\section{Introduction}

It is well known that the contractive type conditions plays an important role in the study of fixed point theory. The first interesting fixed point results for contractive type mappings was obtained by Banach [1] in 1922. The study on contractive type mappings concerning the existence of fixed points has drawn attentions of many mathematicians in non-linear analysis.

In recent years, many researchers have done much work in metric spaces[2], symmetric spaces[3] and [4], $D$-metric space[5] and [6], $G$-metric space[7], $D^{*}$ metric space[8], complete metric space[9], and so on. Dhage [5] introduced

Received: Ocotber 28, 2015

Published: February 15, 2016

${ }^{\S}$ Correspondence author (c) 2016 Academic Publications, Ltd.

url: www.acadpubl.eu 
the notion of $D$-metric space as a generalization of ordinary metric functions in 1992. Later on, joint with Sims, Mustafa demonstrated that most of the claims concerning the fundamental topological properties of $D$-metric spaces are incorrect [10] and [11]. For this reason, they introduced a new notion of generalized metric space which is called $G$-metric space in 2006. Then, Many mathematicians such as Shatanawi [12] and Mustafa [7] proved some fixed point theorems under certain contractive conditions in $G$-metric space. At the same time Sanodia et al.[13] proved a fixed point theorem for single mapping in the $G$-metric space. Later, someone point out that a $D$-metric need not be a continuous function of its variables, in order to overcome this problem, Shaban Sedghi [14] modified the notion of $D$-metric space and defined the notion of $D^{*}$ metric space in 2007. Then, Aage-Salunke [15] proved that the generalized in $D^{*}$-metric spaces by replacing the real numbers with an ordered Banach space and defined $D^{*}$-cone metric spaces and showed the topological properties.

In this paper, we will first introduce the notion of $D_{1}$-space which is weaker than the $D$-metric space and $G$-metric space. Then in $D_{1}$-space we give a theorem on the unique existence of the common fixed points. These results improve many common fixed point theorems in the $D$-metric spaces and $G$ metric spaces. Finally, in the $D_{1}$-space, we give the common fixed point theorem on certain mappings which satisfy some general contractive conditions.

\section{Preliminaries}

In this section, we introduce some basic definitions which will be used throughout this paper.

In 1992, Bapure Dhage introduced a new class of generalized metrics, called $D$-metrics, in his $\mathrm{PhD}$ thesis. The definition of $D$-metric space is stated as follows.

Definition 1. [5] Let $X$ be a non-empty set and let $R$ denote the real numbers. If a function $D: X \times X \times X \rightarrow R^{+}$satisfies the following properties:

$$
\begin{aligned}
& A(0) D(x, y, z)=0 \text { if and only if } \quad x=y=z ; \\
& A(1) D(x, y, z)=D(x, z, y)=\cdots \quad(\text { symmetry in all three variables); } \\
& A(2) D(x, y, z) \leq D(x, y, a)+D(x, a, z)+D(a, y, z), \text { for all } \\
& \quad x, y, z, a \in X
\end{aligned}
$$

then we call it called a $D$-metric and the pair $(X, D)$ is called a $D$-metric space. 
However, most of the claims concerning the fundamental topological properties of $D$-metric spaces are incorrect (see[10, 11]). In 2006, Mustafa and Sims introduced a new structure of generalized metric spaces (see [7]), which are called $G$-metric spaces. The definition of $G$-metric space is stated as follows.

Definition 2. [7] Let $X$ be a non-empty set. Then a function $G: X \times$ $X \times X \rightarrow R^{+}$is a $G$-metric if it satisfies the axioms (A0) and (A1), as well as (A2) replaced by the following axioms:

$$
\begin{array}{ll}
A(3) \quad 0<G(x, x, y) \text { for all } x, y \in X, \text { with } x \neq y \\
A(4) \quad G(x, x, y) \leq G(x, y, z), \text { for all } x, y, z \in X, \text { with } z \neq y \\
A(5) \quad G(x, y, z) \leq G(x, a, a)+G(a, y, z), \text { for all } x, y, z, a \in X \\
\\
\text { (rectangle inequality). }
\end{array}
$$

The pair $(X, G)$ is then called a $G$-metric space.

Later, Shaban Sedghi [14] modified the notion of $D$-metric space and defined the notion of $D^{*}$-metric spaces. The definition of $D^{*}$-metric space is stated as follows.

Definition 3. [14] Let $X$ be a non-empty set. Then a function $D^{*}$ : $X \times X \times X \rightarrow R^{+}$is a $D^{*}$-metric if it satisfies the axioms (A0) and (A1), as well as (A2) replaced by the axioms:

$$
A(6) \quad D^{*}(x, y, z) \leq D^{*}(x, y, a)+D^{*}(a, z, z), \text { for all } x, y, z, a \in X \text {. }
$$

The pair $\left(X, D^{*}\right)$ is then called a $D^{*}$-metric space.

In what follows, we will first introduce a new notion, named as $D_{1}$-space, which is weaker than the notions of $D$-metric space, $G$-metric space and $D^{*}$ metric space. The definition of $D_{1}$ - space is stated as follows.

Definition 4. Let $X$ be a non-empty set. If a function $d: X \times X \times X \rightarrow R^{+}$ satisfying the axiom:

$$
A(0) d(x, y, z)=0 \text { if and only if } x=y=z \text {, }
$$

then the pair $(X, d)$ is called a $D_{1}$-space. 
Next, we give an example of $D^{*}$-space.

Example 5. Let $X=R^{+}, d: X \times X \times X \rightarrow[0,+\infty)$ as follows:

(1) $d(x, y, z)=0$, where $x=y=z$;

(2)while $\max \{|x-y|,|y-z|,|z-x|\} \neq 0$,

$$
\left\{\begin{array}{l}
d(x, y, z)=\max \{y, z\}, \text { where } x=0 \\
d(x, y, z)=6, \quad x=1, y=2, z=3 \\
d(x, y, z)=x, \quad \text { Others }
\end{array}\right.
$$

Clearly, $d(0,1,2)=2 \neq 1=d(1,0,2)$, that $d$ is not symmetry, we get $(X, d)$ is neither $G$-metric space nor $D^{*}$-metric space. Since, $d(1,2,3)=6>$ $5=d(1,2,0)+d(1,0,2)+d(0,1,3)$, then $(X, d)$ also does not $D$-metric space. However, through the above analysis, we have $(\mathrm{X}, \mathrm{d})$ is a $D_{1}$-space.

Definition 6. Let $f$ and $g$ be two self-mappings on $D_{1}$-space $(X, d)$. Then $f$ and $g$ are called converse commuting self-mappings if for all $x \in X$, $f g x=g f x$ implies $f x=g x$.

Definition 7. Let $f$ and $g$ be two self-mappings on a non-empty set $X$ on

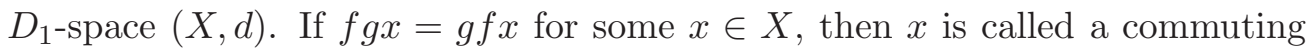
point.

\section{Main Result}

In this section we will give the main result.

Theorem 8. Let $(X, d)$ be a $D_{1}$-space and let $f$ and $g$ be two converse commuting self-maps which have a commuting point. Suppose that for points $x, y, z \in X$, satisfy the condition

$$
d(g x, g y, g z) \leq a_{1} d(f z, f y, g x)+a_{2} d(g z, f y, g x)+a_{3} d(g z, g y, g x)
$$

as well as $a_{1}, a_{2}, a_{3} \in R^{+}$and $0<a_{1}+a_{2}+a_{3}<1$. Then $f$ and $g$ admit a unique common fixed point. 
Proof. We assume that $f$ and $g$ have a commuting point $u$, then $f g u=g f u$. Since $f$ and $g$ are two converse commuting self-maps, then $f f u=f g u=g f u=$ $g g u$. We plan to prove that $g u$ is the fixed point of $g$. In fact, assume that $g u$ is not the fixed point of $g$. Then we have $g g u \neq g u$ as well as

$$
d(g u, g u, g g u)>0 \text { and } d(g g u, g u, g u)>0 .
$$

From (1), there are

$$
\begin{aligned}
d(g u, g u, g g u) \leq & a_{1} d(f g u, f u, g u)+a_{2} d(g g u, f u, g u) \\
& +a_{3} d(g g u, g u, g u) \\
= & \left(a_{1}+a_{2}+a_{3}\right) d(g g u, g u, g u) \\
< & d(g g u, g u, g u)
\end{aligned}
$$

and

$$
\begin{aligned}
d(g g u, g u, g u) \leq & a_{1} d(f u, f u, g g u)+a_{2} d(g u, f u, g g u) \\
& +a_{3} d(g u, g u, g g u) \\
= & \left(a_{1}+a_{2}+a_{3}\right) d(g u, g u, g g u) \\
< & d(g u, g u, g g u)
\end{aligned}
$$

This is a contradiction. It then follows $g g u=g u$ and thus $g u$ is the fixed point of $g$. Since $f g u=f f u=g f u=g g u=g u$, then $g u$ is the fixed point of $f$. Consequently, $f u=g u$ is the common fixed point of $f$ and $g$.

Now we show that $f$ and $g$ have a unique point of coincidence. Assume that there exists a point $v \in X$ such that $f v=g v=v$. From (1), we have

$$
\begin{aligned}
d(u, u, v) & =d(g u, g u, g v) \\
& \leq a_{1} d(f v, f u, g u)+a_{2} d(g v, f u, g u)+a_{3} d(g v, g u, g u) \\
& =\left(a_{1}+a_{2}+a_{3}\right) d(v, u, u) \\
& <d(v, u, u)
\end{aligned}
$$

and

$$
\begin{aligned}
d(v, u, u) & =d(g v, g u, g u) \\
& \leq a_{1} d(f u, f u, g v)+a_{2} d(g u, f u, g v)+a_{3} d(g u, g u, g v) \\
& =\left(a_{1}+a_{2}+a_{3}\right) d(u, u, v) \\
& <d(u, u, v) .
\end{aligned}
$$

This is a contradiction and hence $v=u$. Thus we finish the proof. 
Now we give an example to support our results.

Example 9. Let $X=\{1,2,3,4\}$, and let $d: X \times X \times X \rightarrow[0,+\infty)$ be a mapping satisfies the following condition for all $x, y, z \in X$ :

(1) $d(x, y, z)=0$, where $x=y=z$;

$(2)\left\{\begin{aligned} d(1,1,2) & =\frac{4}{3} ; \\ d(1,2,1) & =d(2,1,1)=d(2,2,1)=d(2,1,2)=d(1,2,2) \\ & =d(1,2,4)=d(1,4,3)=1 ; \\ d(4,2,3) & =d(1,3,4)=d(1,4,2)=d(4,3,2)=d(2,3,4) \\ & =d(2,4,1)=d(4,3,1)=1 ;\end{aligned}\right.$

(3) $d(x, y, z)=8$, Others.

Clearly, $d(1,1,2) \neq d(1,2,1)$, and $d(1,2,3)>d(1,2,4)+d(1,4,3)+d(4,2,3)$, then $(X, d)$ is a $D_{1}-$ space.

Consider mappings $f, g: X \rightarrow X$, by

$$
\begin{gathered}
f(1)=1, f(2)=3, f(3)=3, f(4)=3 \\
g(1)=1, g(2)=1, g(3)=2, g(4)=2 .
\end{gathered}
$$

Let $a_{1}=a_{2}=a_{3}=\frac{1}{4}$, we can get $0<a_{1}+a_{2}+a_{3}<1$. Since $f g(1)=$ $g f(1)=f(1)=g(1)$, then $f$ and $g$ be two converse commuting self-maps which have commuting point $x=1$, so we will verify the contraction condition(1). It have six cases to be considered as following.

Case 1. $d(g x, g y, g z)=d(1,1,2)$, where $x=1$ or $x=2, y=1$ or $y=2$, $z=3$ or $z=4$. We can get $f z=3, g x=1$, then

$$
\begin{aligned}
d(g x, g y, g z)=d(1,1,2)= & \frac{4}{3} \\
< & \frac{1}{4} \times 8 \\
= & \frac{1}{4} d(3, f y, 1) \\
= & \frac{1}{4} d(f z, f y, g x) \\
< & a_{1} d(f z, f y, g x)+a_{2} d(g z, f y, g x) \\
& +a_{3} d(g z, g y, g x) ; \\
&
\end{aligned}
$$

thus, the inequality(1) holds. 
Case 2. $d(g x, g y, g z)=d(1,2,1)$, where $x=1$ or $x=2, y=3$ or $y=4$, $z=1$ or $z=2$. We can get $f y=3, g x=1, g z=1$, then

$$
\begin{aligned}
d(g x, g y, g z)=d(1,2,1)= & 1 \\
< & \frac{1}{4}(8+8) \\
= & \frac{1}{4}(d(f z, 3,1)+d(1,3,1)) \\
= & \frac{1}{4}(d(f z, f y, g x)+d(g z, f y, g x)) \\
< & a_{1} d(f z, f y, g x)+a_{2} d(g z, f y, g x) \\
& +a_{3} d(g z, g y, g x),
\end{aligned}
$$

thus, the inequality(1) holds.

Case 3. $d(g x, g y, g z)=d(2,1,1)$, where $x=3$ or $x=4, y=1$ or $y=2$, $z=1$ or $z=2$. We have the four cases as following:

Case 3.1. For $x=3$ or $x=4, y=1, z=1$. we can get $g x=2, f y=f z=$ $g y=g z=1$, then

$$
\begin{aligned}
d(g x, g y, g z)=d(2,1,1)= & 1 \\
= & \frac{1}{4}\left(\frac{4}{3}+\frac{4}{3}+\frac{4}{3}\right) \\
= & \frac{1}{4}(d(1,1,2)+d(1,1,2)+d(1,1,2)) \\
= & \frac{1}{4}(d(f z, f y, g x)+d(g z, f y, g x) \\
& +d(g z, g y, g x)) \\
= & a_{1} d(f z, f y, g x)+a_{2} d(g z, f y, g x) \\
& +a_{3} d(g z, g y, g x),
\end{aligned}
$$

thus, the inequality(1) holds.

Case 3.2. For $x=3$ or $x=4, y=1, z=2$. we can get $g x=2, f y=$ $g y=g z=1, f z=3$, then

$$
\begin{aligned}
d(g x, g y, g z)=d(2,1,1) & =1 \\
& <\frac{1}{4} \times 8 \\
& =\frac{1}{4} d(3,1,2) \\
& =\frac{1}{4} d(f z, f y, g x)
\end{aligned}
$$




$$
\begin{aligned}
& <a_{1} d(f z, f y, f x)+a_{2} d(g z, f y, g x) \\
& \quad+a_{3} d(g z, g y, g x)
\end{aligned}
$$

thus, the inequality(1) holds.

Case 3.3. For $x=3$ or $x=4, y=2, z=1$, similar to Case 3.2, the inequality (1) holds.

Case 3.4. For $x=3$ or $x=4, y=2, z=2$, similar to Case 3.2, the inequality(1) holds.

Case 4. $d(g x, g y, g z)=d(2,2,1)$, where $x=3$ or $x=4, y=3$ or $y=4$, $z=1$ or $z=2$. We can get $g x=g y=2, f y=3, g z=1$, then

$$
\begin{aligned}
d(g x, g y, g z)=d(2,2,1) & =1 \\
& <\frac{1}{4}(8+8) \\
& =\frac{1}{4}(d(f z, 3,2)+d(1,3,2)) \\
& =\frac{1}{4}(d(f z, f y, g x)+d(g z, f y, g x)) \\
& <a_{1} d(f z, f y, g x)+a_{2} d(g z, f y, g x) \\
& +a_{3} d(g z, g y, g x),
\end{aligned}
$$

thus, the inequality (1) holds.

Case 5. $d(g x, g y, g z)=d(2,1,2)$, where $x=3$ or $x=4, y=1$ or $y=2$, $z=3$ or $z=4$. We can get $g x=g z=2, f z=3, g y=1$, then

$$
\begin{aligned}
d(g x, g y, g z)=d(2,1,2)= & 1 \\
< & \frac{1}{4} \times 8 \\
= & \frac{1}{4} d(3, f y, 2) \\
= & \frac{1}{4} d(f z, f y, g x) \\
< & a_{1} d(f z, f y, g x)+a_{2} d(g z, f y, g x) \\
& +a_{3} d(g z, g y, g x),
\end{aligned}
$$

thus, the inequality(1) holds.

Case 6. $d(g x, g y, g z)=d(1,2,2)$, where $x=1$ or $x=2, y=3$ or $y=4$, $z=3$ or $z=4$. We can get $f y=f z=3, g y=g z=2, g x=1$, then

$$
d(g x, g y, g z)=d(1,2,2)=1
$$




$$
\begin{aligned}
< & \frac{1}{4}(8+8) \\
= & \frac{1}{4}(d(3,3,1)+d(2,3,1)) \\
= & \frac{1}{4}(d(f z, f y, g x)+d(g z, f y, g x)) \\
< & a_{1} d(f z, f y, g x)+a_{2} d(g z, f y, g x) \\
& +a_{3} d(g z, g y, g x),
\end{aligned}
$$

thus, the inequality(1) holds.

Therefore, we showed that the contraction condition(1) is satisfied in all cases. Thus by the Theorem 8 , we can get $f$ and $g$ have a unique common fixed point $x=1$.

Theorem 10. Let $(X, d)$ be a $D_{1}$-space and let $f_{1}, f_{2}, g_{1}, g_{2}$ be four selfmappings. Also we let $\left(f_{1}, f_{2}\right)$ and $\left(g_{1}, g_{2}\right)$ be a pair of converse commuting self-mappings which have a commuting point. Suppose that for $x, y, z \in X$ with $d\left(f_{2} x, f_{2} y, g_{2} z\right) \neq 0$, satisfy the condition

$$
\begin{aligned}
d\left(f_{2} x, f_{2} y, g_{2} z\right) \leq & a_{1} d\left(g_{1} z, f_{1} y, f_{2} x\right)+a_{2} d\left(g_{2} z, f_{1} y, f_{2} x\right) \\
& +a_{3} d\left(g_{2} z, f_{2} y, f_{2} x\right)+a_{4} d\left(f_{1} y, f_{2} y, f_{2} x\right) \\
& +a_{5} d\left(g_{1} z, f_{2} y, f_{2} x\right) .
\end{aligned}
$$

Additionally, we suppose that for $x, y, z \in X$ with $d\left(g_{1} x, f_{1} y, f_{1} z\right) \neq 0$, satisfy the condition

$$
\begin{aligned}
d\left(g_{1} x, f_{1} y, f_{1} z\right) \leq & a_{1} d\left(f_{2} z, f_{2} y, g_{1} x\right)+a_{2} d\left(f_{1} z, f_{2} y, g_{1} x\right) \\
& +a_{3} d\left(f_{1} z, f_{1} y, g_{1} x\right)+a_{4} d\left(f_{2} y, f_{1} y, f_{1} z\right) \\
& +a_{5} d\left(f_{2} z, f_{1} y, g_{1} x\right)
\end{aligned}
$$

as well as $0<a_{1}+a_{2}+a_{3}+a_{5}<1$. Then the four mappings $f_{1}, f_{2}, g_{1}, g_{2}$ have a unique common fixed point.

Proof. Suppose that the pair $\left(f_{1}, f_{2}\right)$ have a commuting point $u$ and $\left(g_{1}, g_{2}\right)$ have a commuting point $v$. Then there are

$$
f_{1} f_{2} u=f_{2} f_{1} u \quad \text { and } \quad g_{1} g_{2} v=g_{2} g_{1} v .
$$

Since $\left(f_{1}, f_{2}\right)$ and $\left(g_{1}, g_{2}\right)$ are pairs of converse commuting self-maps, it clearly follows

$$
f_{1} f_{2} u=f_{2} f_{1} u=f_{1} f_{1} u=f_{2} f_{2} u \quad \text { and } \quad g_{1} g_{2} v=g_{2} g_{1} v=g_{1} g_{1} v=g_{2} g_{2} v .
$$


We claim that $f_{2} u=g_{2} v$. If $f_{2} u \neq g_{2} v$, then there are $d\left(f_{2} u, f_{2} u, g_{2} v\right)>0$ and $d\left(g_{2} v, f_{2} u, f_{2} u\right)>0$. From (2) and (3), we have

$$
\begin{aligned}
d\left(f_{2} u, f_{2} u, g_{2} v\right) \leq & a_{1} d\left(g_{1} v, f_{1} u, f_{2} u\right)+a_{2} d\left(g_{2} v, f_{1} u, f_{2} u\right) \\
& +a_{3} d\left(g_{2} v, f_{2} u, f_{2} u\right)+a_{4} d\left(f_{1} u, f_{2} u, f_{2} u\right) \\
& \quad+a_{5} d\left(g_{1} v, f_{2} u, f_{2} u\right) \\
= & \left(a_{1}+a_{2}+a_{3}+a_{5}\right) d\left(g_{2} v, f_{2} u, f_{2} u\right) \\
< & d\left(g_{2} v, f_{2} u, f_{2} u\right)
\end{aligned}
$$

and

$$
\begin{aligned}
d\left(g_{2} v, f_{2} u, f_{2} u\right)= & d\left(g_{1} v, f_{1} u, f_{1} u\right) \\
\leq & a_{1} d\left(f_{2} u, f_{2} u, g_{1} v\right)+a_{2} d\left(f_{1} u, f_{2} u, g_{1} v\right) \\
& +a_{3} d\left(f_{1} u, f_{1} u, g_{1} v\right)+a_{4} d\left(f_{2} u, f_{1} u, f_{1} u\right) \\
& \quad+a_{5} d\left(f_{2} u, f_{1} u, g_{1} v\right) \\
= & \left(a_{1}+a_{2}+a_{3}+a_{5}\right) d\left(f_{2} u, f_{2} u, g_{2} v\right) \\
< & d\left(f_{2} u, f_{2} u, g_{2} v\right) .
\end{aligned}
$$

This is a contradiction and thus $f_{2} u=g_{2} v$.

Now we claim that $f_{2} u$ is the fixed point of $f_{2}$, that is $f_{2} f_{2} u=f_{2} u$. In fact, if $f_{2} f_{2} u \neq f_{2} u$, then there are

$$
d\left(f_{2} f_{2} u, f_{2} f_{2} u, f_{2} u\right)>0 \quad \text { and } \quad d\left(f_{2} u, f_{2} f_{2} u, f_{2} f_{2} u\right)>0 .
$$

From (2) and (3), we have

$$
\begin{aligned}
d\left(f_{2} f_{2} u, f_{2} f_{2} u, f_{2} u\right)= & d\left(f_{2} f_{2} u, f_{2} f_{2} u, g_{2} v\right) \\
\leq & a_{1} d\left(g_{1} v, f_{1} f_{2} u, f_{2} f_{2} u\right)+a_{2} d\left(g_{2} v, f_{1} f_{2} u, f_{2} f_{2} u\right) \\
& +a_{3} d\left(g_{2} v, f_{2} f_{2} u, f_{2} f_{2} u\right)+a_{4} d\left(f_{1} f_{2} u, f_{2} f_{2} u, f_{2} f_{2} u\right) \\
& +a_{5} d\left(g_{1} v, f_{2} f_{2} u, f_{2} f_{2} u\right) \\
= & \left(a_{1}+a_{2}+a_{3}+a_{5}\right) d\left(g_{2} v, f_{2} f_{2} u, f_{2} f_{2} u\right) \\
< & d\left(f_{2} u, f_{2} f_{2} u, f_{2} f_{2} u\right)
\end{aligned}
$$

and

$$
\begin{aligned}
d\left(f_{2} u, f_{2} f_{2} u, f_{2} f_{2} u\right)= & d\left(g_{1} v, f_{1} f_{1} u, f_{1} f_{1} u\right) \\
\leq & a_{1} d\left(f_{2} f_{1} u, f_{2} f_{1} u, g_{1} v\right)+a_{2} d\left(f_{1} f_{1} u, f_{2} f_{1} u, g_{1} v\right) \\
& +a_{3} d\left(f_{1} f_{1} u, f_{1} f_{1} u, g_{1} v\right)+a_{4} d\left(f_{2} f_{1} u, f_{1} f_{1} u, f_{1} f_{1} u\right)
\end{aligned}
$$




$$
\begin{aligned}
& +a_{5} d\left(f_{2} f_{1} u, f_{1} f_{1} u, g_{1} v\right) \\
= & \left(a_{1}+a_{2}+a_{3}+a_{5}\right) d\left(f_{2} f_{2} u, f_{2} f_{2} u, g_{1} v\right) \\
< & d\left(f_{2} f_{2} u, f_{2} f_{2} u, f_{2} u\right) .
\end{aligned}
$$

It follows $f_{2} f_{2} u=f_{2} u$ and then $f_{2} u$ is the fixed point of $f_{2}$.

In a similar way we can show that $g_{2} g_{2} v=g_{2} v$. Now we have obtained

$$
g_{1} g_{2} v=g_{2} g_{1} v=g_{2} v \quad \text { and } \quad f_{2} u=f_{1} f_{2} u=f_{2} f_{2} u \text {. }
$$

It follows that $f_{2} u=g_{2} v$ is the common fixed point of $f_{1}, f_{2}, g_{1}, g_{2}$.

Now we prove that $f_{1}, f_{2}, g_{1}, g_{2}$ have a unique common fixed point. Assume that there exist two points $s, t \in X$ such that

$$
s=f_{2} s=g_{1} s=g_{2} s \text { and } f_{1} t=f_{2} t=g_{1} t=t .
$$

It follows

$$
d(s, s, t)<d(t, s, s) \text { and } d(t, s, s)<d(s, s, t)
$$

and hence $s=t$. Thus the uniqueness is proved and the proof is finished.

Remark. If $d$ is a symmetric mapping, then Theorem 10 can hold under the condition only (2) or (3).

Now we give an example to support our results.

Example 11. Let $X=\{1,2,3,4,5\}$, and let $d: X \times X \times X \rightarrow[0,+\infty)$ be a mapping satisfies the following condition for all $x, y, z \in X$ :

(1) $d(x, y, z)=0$, where $x=y=z$;

$$
\left\{\begin{aligned}
d(2,2,1) & =d(2,1,1)=d(1,2,1)=d(1,1,4)=d(2,2,4)=1 \\
d(1,2,4) & =d(2,1,4)=d(1,3,3)=d(1,3,1)=d(1,1,3) \\
& =d(5,1,1)=1 \\
d(5,3,3) & =d(5,1,3)=d(5,3,1)=d(1,2,4)=d(1,4,3) \\
& =d(4,2,3)=1
\end{aligned}\right.
$$

(3) $d(x, y, z)=5$, Others.

Clearly, $d(2,1,1) \neq d(1,1,2)$, and $d(1,2,3)>d(1,2,4)+d(1,4,3)+d(4,2,3)$, then $(X, d)$ is a $D_{1}-$ space.

Consider mappings $f, g: X \rightarrow X$, by

$$
\begin{aligned}
& f_{1}(1)=1, f_{1}(2)=3, f_{1}(3)=3, f_{1}(4)=3, f_{1}(5)=3 ; \\
& f_{2}(1)=1, f_{2}(2)=2, f_{2}(3)=2, f_{2}(4)=2, f_{2}(5)=2 ;
\end{aligned}
$$




$$
\begin{aligned}
& g_{1}(1)=1, g_{1}(2)=5, g_{1}(3)=5, g_{1}(4)=5, g_{1}(5)=5 \\
& g_{2}(1)=1, g_{2}(2)=4, g_{2}(3)=4, g_{2}(4)=4, g_{2}(5)=4
\end{aligned}
$$

Let $a_{1}=a_{2}=a_{3}=a_{4}=a_{5}=\frac{1}{5}$, we can get $0<a_{1}+a_{2}+a_{3}+a_{5}<1$. Since $f_{1} f_{2}(1)=f_{2} f_{1}(1), f_{1}(1)=f_{2}(1)$, then $f_{1}$ and $f_{2}$ be two converse commuting self-maps which have commuting point $x=1 ; g_{1} g_{2}(1)=g_{2} g_{1}(1), g_{1}(1)=g_{2}(1)$, then $g_{1}$ and $g_{2}$ be two converse commuting self-maps which have commuting point $x=1$, so we will verify the contraction condition(2)and(3). It have the following cases to be considered.

Case 3.1. $d\left(f_{2} x, f_{2} y, g_{2} z\right)=d(2,2,1)$, where $x=2$ or $x=3$ or $x=4$ or $x=5, y=2$ or $y=3$ or $y=4$ or $y=5, z=1$. We can get $f_{1} y=3, f_{2} y=$ $2, f_{2} x=2$, then

$$
\begin{aligned}
d\left(f_{2} x, f_{2} y, g_{2} z\right)= & d(2,2,1)=1=\frac{1}{5} \times 5=\frac{1}{5} d(3,2,2) \\
= & a_{4} d\left(f_{1} y, f_{2} y, f_{2} x\right) \\
< & a_{1} d\left(g_{1} z, f_{1} y, f_{2} x\right)+a_{2} d\left(g_{2} z, f_{1} y, f_{2} x\right) \\
& +a_{3} d\left(g_{2} z, f_{2} y, f_{2} x\right)+a_{4} d\left(f_{1} y, f_{2} y, f_{2} x\right) \\
& +a_{5} d\left(g_{1} z, f_{2} y, f_{2} x\right)
\end{aligned}
$$

thus, the inequality (2) holds.

The following proof similar to Example 9, we can no longer prove in detail. Therefore, we showed that the contraction condition $(2),(3)$ is satisfied in all cases. Thus by the Theorem 10, we can get $f$ and $g$ have a unique common fixed point $x=1$.

\section{Acknowledgments}

This project is supported by NSFC (NO.11101161 and NO.11261062) and China Postdoctoral Science Foundation funded project(NO.2014M562129).

\section{References}

[1] S. Banach, Sur les opérations dans les ensembles abstraits et leur application auxéquations int égrales, Fundam. Math., 3 (1922), 133-181.

[2] R. P. Pant, Common fixed point theorems for contractive maps, J. Math. Anal. Appl., 22 (1998), 252-258.

[3] T. L. Hicks, B. E. Rhoades, Fixed point theory in symmetric spaces with Applications to probabilistic spaces, Nonlinear Analysis., 36 (1993), 331-334. 
[4] M. Asmri, D. E. Moutawakil, Common fixed points under strict contractive conditions in symmetric space, Applied Mathematics E-notes., 3 (2003), 156-162.

[5] B. C. Dhage, Generalized metric space and mapping with fixed point, Bull. C Cal. Math. Soc., 84 (1992), 329-336.

[6] B. C. Dhage, Generalized metric space and topological structure I, An. Stiint. Univ. Al. I. Cuza Iasi. Mat.(N.S)., (2000), 3-24.

[7] Z. Mustafa, B. Sims, A new approach to generalized metric spaces, J. Nonlinear Convex Anal., 7 (2006), 289-297.

[8] V. Sachin, S. M. Bedre, B. S. Deasle, B. Ramakant, Fixed and common fixed point theorems in $D^{*}$-metric space, International J. Math., 4 (2012), 309-328.

[9] D. F. Xia, M. F. Fu, B. Jiang, Two Self-mapping Common Fixed Points for Symmetric Sets and Complete Metric Space, Adv. Math., 36 (2007), 415-420.

[10] Z. Mustafa, B. Sims, Some Remarks Concerninig D-metric space, Proceedings of the Internatinal Conferences on Fixed Point Theorey and Applications, Valenci(Spain)., July (2003), 189-198.

[11] S. V. R. Naidu, K. P. R. Rao, N. S. Rao, On the concepts of balls in a $D$-metric space, Int. J. Math. and Math. Sci., 1 (2005), 133-141.

[12] S. P. Singh, Some results on fixed point theorems, Yokahama Math. J., 17 (1969), 61-64.

[13] V. M. Sehgal, On fixed and periodic points for a class of mappings, J. London Math. Soc., 5 (1972), 571-576.

[14] S. Sedghi, N. Shobe, H. Zhou, A common fixed point theorem in $D^{*}$-metric spaces, Fixed Point Theory and Appl., 01 (2007), 1-14.

[15] C. T. Aage, J. N. Solunke, Some fixed point theorems in generalized $D^{*}$-metric space, Applied Saci., 12 (2010), 1-13. 
